\title{
Parallel Propagation Effects on the Type 1 Electrojet Instability
}

\author{
S. L. Ossakow, K. Papadopoulos, J. Orens, and T. Coffey
}

Naval Research Laboratory, Washington, D. C. 20375

\begin{abstract}
The Farley-Buneman instability has been extended to consider higher-frequency shorter-wavelength modes (thus including finite Debye length effects), and these modes are allowed to propagate with a component parallel to the magnetic field $\left(k_{11} \neq 0\right)$. When the current is driven sufficiently hard (drift speeds in the range 2-3 times the ion thermal velocity $v_{t}$ ), the growth rates of these modes maximize slightly away from the perpendicular to the magnetic field, and thus the importance of $k_{||} \neq 0$ is shown. Although the wavelengths of these maximum growing modes are in the regime of tens of centimelers, the phase velocities are closer to the ion thermal velocity than those modes propagating at $90^{\circ}\left(k_{11}=0\right)$. Maximum growth rates of off-angle propagation for different densities and collision lrequencies are shown. Also, growth rates of unstable waves in the radar regime $(1-10 \mathrm{~m})$ are shown for drift velocities $1.5 v_{l}$ and $3 v_{l}$. These also maximize with $k_{11} \neq 0$ and have phase velocities closer to $v_{1}$ than they have for purely perpendicular propagation. In all cases considered the phase velocity of the waves is a rapidly decreasing function of angle as one moves away from pure perpendicular propagation.
\end{abstract}

Observations of backscattered radar signals from the equatorial electrojet have provided the impetus for substantial theoretical research on the source of the density fluctuations that can provide the observed enhancements in the received signals. There is general agreement that the source of the density fluctuations is an electron current across the magnetic field produced by $\mathrm{E} \times \mathrm{B}$ type particle drifts due to the disparity in the electron-neutral and ion-neutral collision frequencies [Farley, 1963a,b]. Since the relative drifts produced are of the order of the ion sound speed $v_{l}$ and since the electron and ion temperatures $T_{e, i}$ are equal, the system would have been linearly stable to ion sound on collisionless time scales. However, for collisional time scales the system becomes unstable to low-frequency long-wavelength modes [Buneman. 1963]. The nature of this instability is purely resistive. That is, the resistive medium extracts energy from the ion drift and transfers it to the negative energy wave (slow wave) associated with the drifting ions.

This instability has been examined first by Farley $[1963 a, b]$ for a kinetic plasma and subsequently by Buneman [1963] in the fluid approximation. In Farley's kinetic description, which neglected finite Debye length effects $\left(k \lambda_{D} \rightarrow 0\right)$, it was shown that the important modes were perpendicular to the magnetic field. Lee et al. [1971] have extended Farley's results by including Debye length effects and found higher-frequency shorter-wavelength instabilities. Their calculation was restricted to modes propagating exactly perpendicular to the magnetic field $\left(k_{||}=0\right)$. Very recently, Lee and Kennel [1973] have considered a simple parallel propagation effect analysis on type 1 instabilities in the fluid limit, and they note that these modes may be more unstable than those that propagate across the magnetic field.

Recent theoretical results [Krall and Liewer, 1971; McBride et al., 1972] have shown that even for a collisionless plasma with $T_{e} \sim T_{i}$, a current perpendicular to the magnetic field produces an instability with small but finite $k_{||}\left(k_{||} / k_{\perp} \sim\right.$ $\left.\left(m_{e} / m_{l}\right)^{1 / 2}\right)$. This instability, usually called the modified twostream instability, is a strong reactive type instability, and nonlinear considerations show that one has to go to the strongturbulence regime for saturation. We therefore feel that an examination of whether and when such modes $\left(k_{\|} \neq 0\right)$ become unstable in the electrojet is necessary before going to the

Copyright $(\mathcal{1} 1975$ by the American Geophysical Union. nonlinear theory, which will correlate the observations with the equatorial microstructure.

In the present note we consider the linear theory of the electrojet instability with finite Debye length effects for modes having a small component parallel to the magnetic field $\left(k_{||} \neq\right.$ $0)$. We focus our attention especially in the parameter regions where the nature of the instability changes from resistive to reactive (for a large range of parameters applicable to the electrojet the instability is probably resistive-inductive rather than purely resistive or purely reactive). We present numerical results relevant to the equatorial and the auroral electrojet and discuss their effect on the radar backscattered spectra. For the convenience of the reader we give a simple physical description of the nature of the electrojet type instability (resistive or reactive) in the appendix.

\section{THEORY}

The starting point of the calculation is the kinetic equation with the number-conserving Bhatnagar-Gross-Krook collision term [Bhatnagar et al., 1954]

$$
\begin{aligned}
\frac{\partial f^{i, e}}{\partial t}+\nabla \cdot \nabla f^{i, e} \pm \frac{e}{m_{i, e}} & \left(\mathrm{E}+\frac{\nabla \times \mathrm{B}}{c}\right) \cdot \nabla_{v} f^{i, e} \\
& =-\nu_{i, e}\left(f^{i, e}-\frac{n^{i, e}}{n_{0}} f_{0}^{i, e}\right)
\end{aligned}
$$

where $i$ and $e$ refer to ions and electrons; $\nu_{i}$ and $\nu_{e}$ are the ionneutral and electron-neutral collision frequencies, respectively, and

$$
n^{i, \bullet}=\int f^{1 \cdot \bullet} d^{3} v
$$

We also utilize Poisson's equation

$$
\nabla \cdot \mathbf{E}=4 \pi e \int\left(f^{i}-f^{e}\right) d^{3} v
$$

and assume

$$
\begin{aligned}
f_{0}{ }^{i} & =\frac{n_{0}}{v_{i}{ }^{3} \pi^{3 / 2}} \exp \left(-\frac{\left(\mathrm{v}-\mathrm{v}_{0}\right)^{2}}{v_{i}{ }^{2}}\right) \\
f_{0} & =\frac{n_{0}}{v_{e}{ }^{3} \pi^{3 / 2}} \exp \left(-\frac{v^{2}}{v_{e}{ }^{2}}\right) \\
f^{i, e} & =f_{0}{ }^{2, e}+f_{1}^{i, e}
\end{aligned}
$$


where $f_{1}$ represents a linear perturbation. The quantities $v_{\imath, e}$ represent the ion and electron thermal velocities, respectively, and $v_{0}$ is the relative drift velocity due to $\mathrm{E} \times \mathrm{B}$ motion (we are in the frame where the electrons are taken to be at rest). The solution of the linearized equations yields the dispersion relation for equal ion and electron temperatures

$$
\begin{aligned}
\left(k \lambda_{D}\right)^{2}+ & {\left[1+\frac{\left(\omega-\mathbf{k} \cdot \mathbf{v}_{0}+i \nu_{2}\right)}{k v_{i}} Z\left(\frac{\omega-\mathbf{k} \cdot v_{0}+i \nu_{i}}{k v_{a}}\right)\right] } \\
\cdot & {\left[1+\frac{i \nu_{i}}{k v_{i}} Z\left(\frac{\omega-\mathbf{k} \cdot \nabla_{0}+i \nu_{i}}{k v_{i}}\right)\right]^{-1} } \\
+ & {\left[1+\frac{\omega+i \nu_{e}}{k_{\|} v_{e}} e^{-} \mu_{e} \sum_{n=-\infty}^{\infty} I_{n}\left(\mu_{e}\right) Z\left(\frac{\omega+i \nu_{e}-n \Omega_{e}}{k_{\|} v_{e}}\right)\right] } \\
& \cdot\left[1+\frac{i \nu_{e}}{k_{\|} v_{e}} e^{-} \mu_{e} \sum_{n=-\infty}^{\infty} I_{n}\left(\mu_{e}\right) Z\left(\frac{\omega+i \nu_{e}-n \Omega_{e}}{k_{\|} v_{e}}\right)\right]^{-1}=0
\end{aligned}
$$

where

$$
\begin{gathered}
\Omega_{0}=\frac{e B}{m_{e} c} \quad \mu_{\theta}=\frac{k_{\perp}{ }^{2} v_{s}{ }^{2}}{2 \Omega_{\theta}{ }^{2}} \quad \lambda_{D}{ }^{2}=\frac{k T_{e}}{4 \pi n_{0} e^{2}} \\
v_{i, \theta}=\left(\frac{2 k T_{i, e}}{m_{i, e}}\right)^{1 / 2} \quad k_{\|}=k \cos \theta \quad \omega=\omega_{r}+i \gamma
\end{gathered}
$$

$Z$ is the plasma dispersion function [Fried and Conte, 1961], and $I_{n}$ are modified Bessel functions. The gyroradius of the ions has been assumed to be large, and $\nu_{l} \gg \Omega_{l}$ (appropriate to electrojet conditions). Further simplification results when we take $\mu_{e}<1, k_{\|} v_{e} \ll \omega$, and $k_{\|} \ll k_{\perp}$, in which case we obtain as the simplified dispersion relation

$$
\begin{aligned}
-\frac{1}{\left(k \lambda_{D}\right)^{2}} & {\left[1+\left(\frac{\omega-k_{\perp} v_{0}+i \nu_{i}}{k v_{i}}\right) z\left(\frac{\omega-k_{\perp} v_{0}+i \nu_{i}}{k v_{i}}\right)\right] } \\
& \cdot\left[1+\frac{i \nu_{i}}{k v_{i}} z\left(\frac{\omega-k_{\perp} v_{0}+i \nu_{i}}{k v_{i}}\right)\right]^{-1} \\
= & 1+\left[\left(\frac{\omega_{p e}}{\Omega_{e}}\right)^{2}\right]\left[1-\frac{i \nu_{e}}{\omega+i \nu_{e}}\right]^{-1} \\
& -\left[\left(\frac{k_{\|}}{k}\right)^{2} \frac{\omega_{p e}^{2}}{\left(\omega+i \nu_{e}\right)^{2}}\right]\left[1-\frac{i \nu_{e}}{\omega+i \nu_{e}}\right]^{-1}
\end{aligned}
$$

where we have taken $v_{0}$ to be perpendicular to the magnetic field $\left(k_{\perp}=k \sin \theta\right)$. The electron contribution (the last two terms) to this dispersion relation are reminiscent of their contribution in the case of the modified two-stream instability [McBride et al., 1972] for a collisionless plasma. Indeed, in the limit $\nu_{i, e} \rightarrow 0$ we recover the dispersion relation applicable for the modified two-stream instability. This instability shows strong growth at angles of the order of $\left(m_{e} / m_{i}\right)^{1 / 2}$ away from perpendicular to the magnetic field and zero growth exactly perpendicular to the magnetic field. Equation (6) can be extracted from the recent work of Schmidt and Gary [1973] in their study of unstable electrostatic waves in a collisional plasma undergoing $\mathbf{E} \times \mathbf{B}$ and density gradient drifts. However, these authors did not look at the peculiarities of wave propagation not exactly perpendicular to the magnetic field. The changing nature of the instability is shown in the appendix.

\section{Discussion OF RESUlts}

We present below the results of a detailed numerical study of the dispersion relation with $k_{\|}$effects for parameters relevant to the electrojet region. Although (6) gives relatively correct results for the long-wavelength $(1-10 \mathrm{~m})$ irregularities observed by the radar where $\mu_{e} \ll 1$, it breaks down for shorter wavelengths, where the effects of the finite electron gyroradius become important. For these reasons we have solved numerically (5) instead of (6). In utilizing (5) the number of modified Bessel functions kept was dictated by the condition that the relative correction be smaller than $10^{-7}$.

The results derived are presented in the ion reference frame so that a direct comparison with the radar observations can be facilitated. Equation (5) was derived in the electron reference frame so that -the results of (6) can be compared directly with the modified two-stream instability.

In the first study of the dispersion relation (5) we have used the following parameters applicable to the electrojet region (which are similar to those used by Farley [1963a, b], Lee et al. [1971], and Schmidt and Gary [1973]);

$$
\begin{aligned}
n_{0} & =3 \times 10^{5} \mathrm{~cm}^{-3} & B_{0} & =0.5 \mathrm{G} \\
T_{0} & =T_{i}=230^{\circ} \mathrm{K} & \nu_{e} & =2.6 \times 10^{4} \mathrm{~s}^{-1} \\
\nu_{\imath} & =1.5 \times 10^{3} \mathrm{~s}^{-1} & m_{\imath} / m_{e} & =5.5 \times 10^{4}
\end{aligned}
$$

The value of $m_{l} / m_{\mathrm{e}}$ corresponds to assuming the dominant ion to be $\mathrm{NO}^{+}$. We have allowed $v_{0}$ to vary from a typical electrojet value of $5.35 \times 10^{4} \mathrm{~cm} / \mathrm{s}$ to a higher value of $1.07 \times 10^{5}$ $\mathrm{cm} / \mathrm{s}$ to observe the effects on the modes that have $k_{11} \neq 0$. Figure 1 shows the cases where $v_{0}=5.35 \times 10^{4} \mathrm{~cm} / \mathrm{s}\left(v_{0} / v_{t}=\right.$ $1.5) ; v_{0}=7.13 \times 10^{4} \mathrm{~cm} / \mathrm{s}\left(v_{0} / v_{l}=2\right) ; v_{0}=8.9 \times 10^{4} \mathrm{~cm} / \mathrm{s}$ $\left(v_{0} / v_{l}=2.5\right) ; v_{0}=1.07 \times 10^{5} \mathrm{~cm} / \mathrm{s}\left(v_{0} / v_{l}=3\right) ;$ and finally, $v_{0} / v_{t}=5.35 \times 10^{4} \mathrm{~cm} / \mathrm{s}$ with $\nu_{e}=\nu_{l}=0$ (i.e., the modified

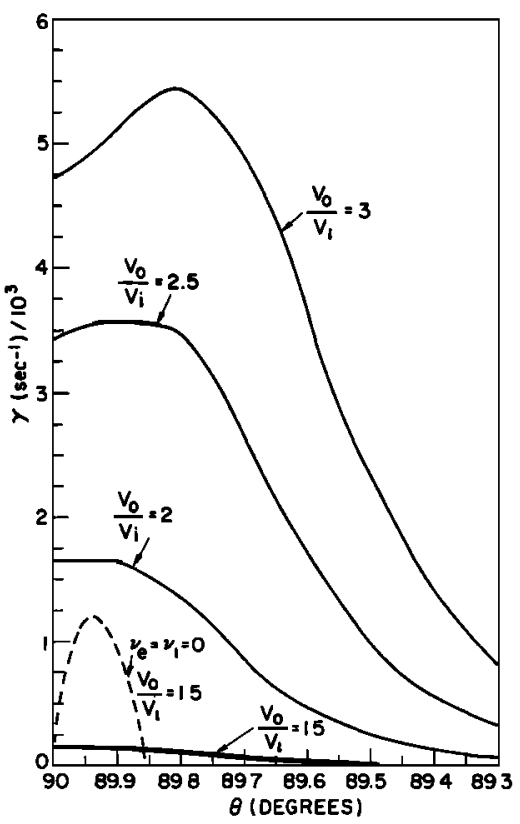

Fig. 1. Plot of growth rate versus the angle $\theta$ that the propagation vector makes with the ambient magnetic field for several values of the drift velocity. Parameters used were $n_{0}=3.5 \times 10^{5} \mathrm{~cm}^{-3}$, $T_{e}=T_{i}=230^{\circ} \mathrm{K}, \nu_{e}=2.6 \times 10^{4} \mathrm{~s}^{-1}, \nu_{i}=1.5 \times 10^{3} \mathrm{~s}^{-1}, m_{i} / m_{e}=$ $5.5 \times 10^{4}$, and $B_{0}=0.5 \mathrm{G}$. For comparison, the case $\nu_{e}=\nu_{i}=0$ (modified two-stream instability) with $v_{0} / v_{i}=1.5\left(v_{i}=3.57 \times 10^{4}\right.$ $\mathrm{cm} / \mathrm{s})$ is shown. 


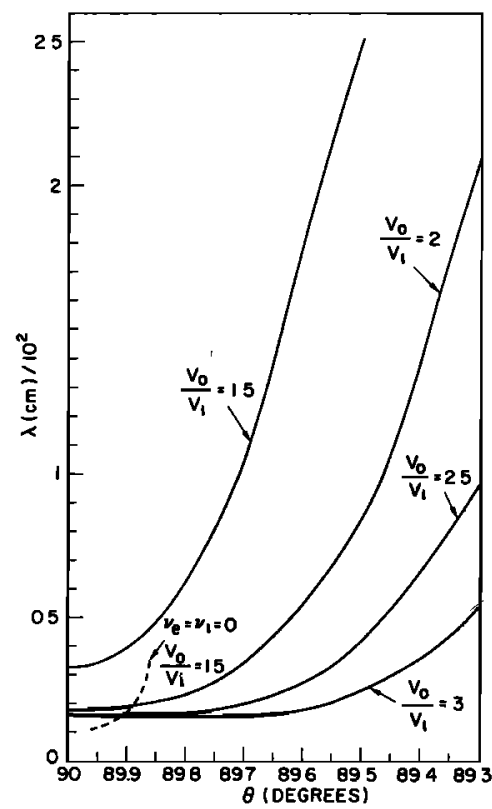

Fig. 2. Wavelength $\lambda$ versus angle $\theta$ corresponding to the unstable wave growths plotted in Figure 1.

two-stream case). The growth rate $\gamma$ (the largest growth at each angle) is plotted against the angle that $\mathbf{k}$ makes with $\mathbf{B}_{\mathbf{0}}$. In the case where $v_{0} / v_{l}=1.5$ the real part of the frequency $\omega_{r}$ (noting that we are now in the ion rest frame) decreases from $9.4 \times 10^{3} \mathrm{~s}^{-1}$ at $90^{\circ}$ to $10^{3} \mathrm{~s}^{-1}$ at $89.5^{\circ}$ (on this scale, growth rates for angles $<89.5^{\circ}$ are too small to plot). Figure 2 shows how, correspondingly, $\lambda=2 \pi / k$ increases from $32 \mathrm{~cm}$ at $90^{\circ}$ to $251 \mathrm{~cm}$ at $89.5^{\circ}$. For this case the largest growth rate occurs at $90^{\circ}$ with a falloff away from this angle. In Figure 3 we note the rapid decrease in phase velocity of the unstable waves as one moves away from $90^{\circ}$. For $v_{0} / v_{t}=1.5, v_{p h} / v_{t}$ goes from 1.36 at $90^{\circ}$ to 1.13 at $89.5^{\circ}$ (the growth rate is 12.8 at $89.5^{\circ}$ compared with 150 at $90^{\circ}$ ). In the curve for the modified twostream instability $\left(\nu_{e}=\nu_{l}=0\right)$ in Figure 1 the behavior is quite different as $\lambda$ increases (see Figure 2$)$ and $\omega_{r}$ decreases $(2.77 \times$ $10^{4}$ to $6.4 \times 10^{3}$ ) as we go from $89.96^{\circ}$ to $89.86^{\circ}$. In this case the growth rate maximizes at $89.94^{\circ}$ and falls off quite rapidly away from this point (the modified two-stream instability having zero growth rate at $90^{\circ}$ ). If we view the $v_{0} / v_{t}=1.5$ curve (Figure 1) with collisions as adding collisions to the modified two-stream case where $\nu_{e} \sim \omega_{r}$ (for modified two-stream case), we see a drastic change in the growth rate and overall behavior of what is known as the modified two-stream instability. As $v_{0}$ is increased, the growth rates both perpendicular (as noted by Lee et al. [1971]) and nonperpendicular to the magnetic field increase substantially (see Figure 1), the frequencies increasing and the wavelengths decreasing (see Figure 2). For $v_{0} / v_{i}=2$ the growth curve is flat to $89.9^{\circ}$ and decreases from theré. However, for $v_{0} / v_{\imath}=2.5$ and 3 the nature of $\gamma$ versus $\theta$ has changed in that the maximum growth rate is no longer at $90^{\circ}$ but has begun to move away; i.e., $k_{11} \neq 0$ modes are becoming important. As we move away from perpendicular, for a given $v_{0} / v_{i}$ the frequency decreases, and the wavelength increases. (For $v_{0} / v_{l}=2$ the range for $\left[\lambda(\mathrm{cm}), \omega_{r}\left(\mathrm{~s}^{-1}\right)\right]$ is $\left[17.4,2.1 \times 10^{4}\right]$ for $90^{\circ}$ to $\left[209,1.3 \times 10^{9}\right]$ for $89.3^{\circ}$, whereas for $v_{0} / v_{i}=2.5$ the corresponding range is $\left[16.5,2.54 \times 10^{4}\right]$ for $90^{\circ}$ to $[96,2.9 \times$ $\left.10^{9}\right]$ for $89.3^{\circ}$, and for $v_{0} / v_{l}=3$ the corresponding range is $\left[17.4,2.7 \times 10^{4}\right]$ for $90^{\circ}$ to $\left[55,5 \times 10^{8}\right]$ for $89.3^{\circ}$.) The severe

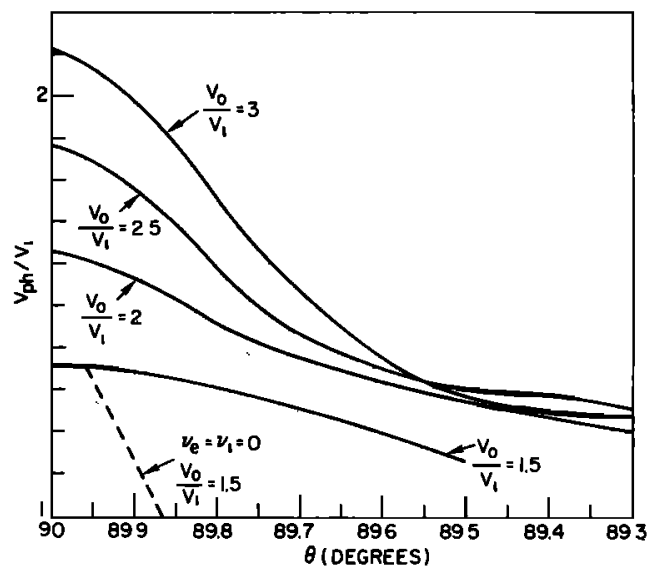

Fig. 3. Phase velocity in units of ion acoustic velocity $\left(v_{p h} / v_{l}\right)$ versus angle $\theta$ for unstable waves plotted in Figure 1 .

decrease in wave phase velocity for $v_{0} / v_{t}=2,2.5$, and 3 is exhibited in Figure 3. Figure 1 shows how the nature of the instability changes as the drift velocity increases.

The effects on the growth rate due to a change in density are shown in Figure 4 . Here we have used $v_{0} / v_{t}=3$ and used the same temperatures, mass ratio, and magnetic field as those used in Figure 1. In going from $n_{0}=3.5 \times 10^{5} \mathrm{~cm}^{-3}$ to $n_{0}=2.3$ $\times 10^{3} \mathrm{~cm}^{-3}$ we note that the growth rate decreases not only at $90^{\circ}$ (as was noted by Lee et al. [1971]) but also away from $90^{\circ}$ because of finite Debye length effects. The frequency $\omega_{r}$ is a decreasing function of angle away from $90^{\circ}$, and the wavelength first decreases slightly and then increases (see Figure 5) for this drift velocity and the density values shown $\left(\left[\lambda(\mathrm{cm}), \omega_{r}\left(\mathrm{~s}^{-1}\right)\right]\right.$ from $90^{\circ}$ to $89.3^{\circ}$ range from $\left[17.4,2.7 \times 10^{4}\right]$ to $\left[55,5 \times 10^{3}\right]$ for $n_{0}=3.5 \times 10^{6} \mathrm{~cm}^{-3}$; from $\left[18.5,2.57 \times 10^{4}\right]$ to $\left[57,4.9 \times 10^{3}\right]$ for $n_{8}=1.16 \times 10^{5} \mathrm{~cm}^{-3}$; from $\left[25,2 \times 10^{4}\right]$ to $\left[60,4.7 \times 10^{9}\right]$ for $n_{0}=2.3 \times 10^{4} \mathrm{~cm}^{-3}$; and from $[60,9.3 \times$ $\left.10^{3}\right]$ to $\left[90,3.5 \times 10^{3}\right]$ for $\left.n_{0}=2.3 \times 10^{3} \mathrm{~cm}^{-3}\right)$. Figure 5 shows that for a given angle the smaller the $n_{0}$ the larger the wavelength of the most unstable wave (note that in Figures 5 and 6 the $n_{0}=1.16 \times 10^{5} \mathrm{~cm}^{-3}$ case has been omitted because of its proximity to the $3.5 \times 10^{5} \mathrm{~cm}^{-3}$ case). Figure 6 depicts the phase velocity versus angle for the most unstable waves. This shows that for a given angle the smaller the $n_{0}$ the larger the phase velocity of the unstable wave. Also we see the rapid

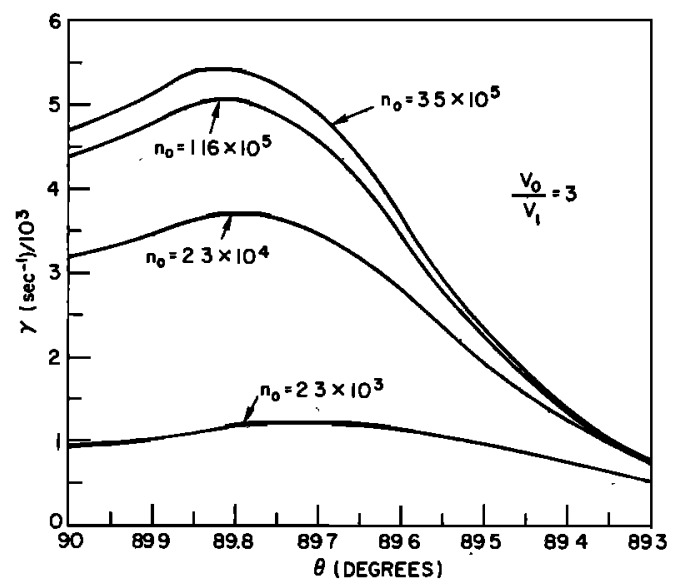

Fig. 4. Growth rate versus angle for several values of the density $n_{0}$ (in units of per centimeter cubed) with the drift velocity $v_{0} / v_{t}=3$. All other parameters are the same as those in Figure 1. 


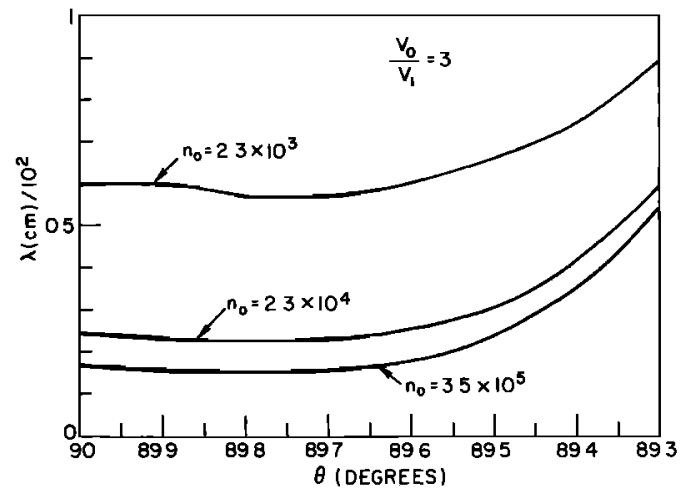

Fig. 5. Wavelength $\lambda$ versus angle $\theta$ corresponding to the unstable waves plotted in Figure 4. The curve corresponding to $n_{0}=1.16 \times 10^{5}$ $\mathrm{cm}^{-3}$ has been omitted because of its proximity to the $n_{0}=3.5 \times 10^{5}$ $\mathrm{cm}^{-3}$ curve.

decrease in phase velocity as we move away from perpendicularity $\left(90^{\circ}\right)$ for fixed $n_{0}$.

Figure 7 shows what effect varying the collision frequencies has on the growth rates (with $v / v_{l}=3, n_{0}=3.5 \times 10^{5}$, and all other parameters the same as those in Figure 1). The canonical frequencies $\left(\nu_{e}=2.6 \times 10^{4} \mathrm{~s}^{-1}\right.$ and $\left.\nu_{i}=1.5 \times 10^{3} \mathrm{~s}^{-1}\right)$ could presumably vary within the vertical region of the electrojet, since at that altitude the neutral scale height is $\sim 10 \mathrm{~km}$. Increasing both the electron and the ion collision frequencies by a factor of 2 over the canonical numbers tends to flatten the growth rate from $90^{\circ}$ to $89.8^{\circ}$ and decrease the off-angle growth rates. The wavelength remains fiat from $90^{\circ}$ to $89.8^{\circ}$ and then increases (see Figure 8), whereas the frequency decreases from $90^{\circ}$ to $89.3^{\circ}$ (the range is $\left[19.6,2.1 \times 10^{4}\right]$ at $90^{\circ}$ to $\left[43,6.7 \times 10^{\circ}\right]$ at $\left.89.3^{\circ}\right)$. Figure 9 shows the decrease in phase velocity as a function of angle. Decreasing the collision frequencies by a factor of 2 over the canonical values results in a more accentuated growth rate peak away from $90^{\circ}$ and enhanced growth rates off angle. In this case the wavelength first decreases slightly and then increases (similar to the canonical case; see Figure 8), whereas the frequency decreases for increasing angle away from $90^{\circ}$ (the range here is [16.8, 3.1 $\left.\times 10^{4}\right]$ at $90^{\circ}$ to $\left[90,3 \times 10^{3}\right]$ at $89.3^{\circ}$ ). For completeness, the limit of zero collision frequency (modified two-stream instability) is shown. (Note that in this case of higher $v_{0} / v_{\imath}$ for the modified two-stream case the growth rate is not only larger

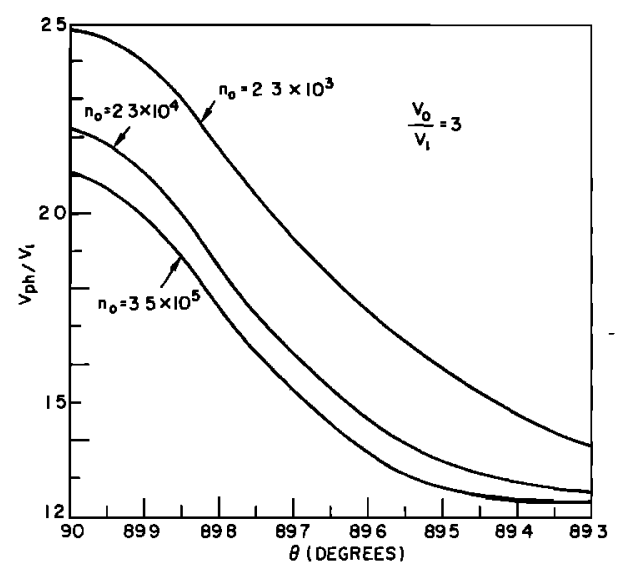

Fig. 6. Phase velocity in units of ion acoustic velocity $\left(v_{p h} / v_{l}\right)$ versus angle $\theta$ for unstable waves plotted in Figure 4 . The $n_{0}=1.16 \times$ $10^{6} \mathrm{~cm}^{-3}$ curve has been omitted because of its proximity to the $n_{0}=$ $3.5 \times 10^{5} \mathrm{~cm}^{-3}$ curve.

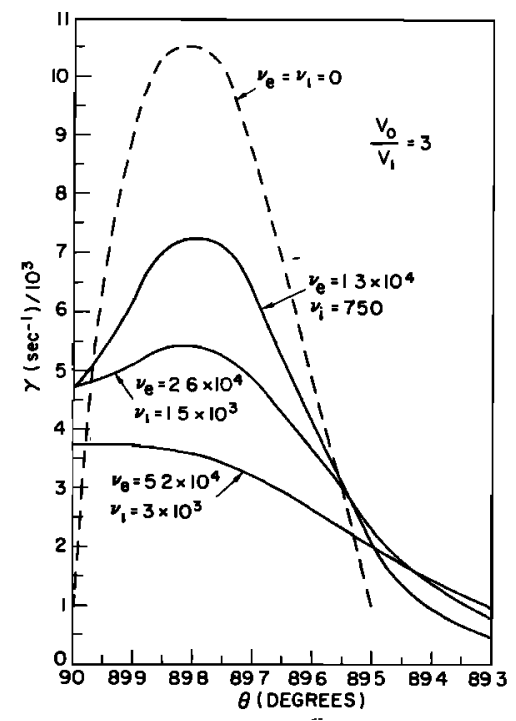

Fig. 7. Growth rate versus angle for several values of the collision frequencies with $v_{0} / v_{l}=3$. All other parameters are the same as those in Figure 1. For comparison, the case with $\nu_{e}=\nu_{t}=0$ and $v_{0} / v_{i}=3$ is also shown.

than that for the modified two-stream instability for $v_{0} / v_{t}=$ 1.5 in Figure 1 but the peak occurs farther away from $90^{\circ}$, and the spread of the curve is larger.) Figure 8 shows that for angles greater than $89.45^{\circ}$ the greater the collision frequency the larger the wavelength of the most unstable wave for a given angle. In contrast, for angles less than $89.45^{\circ}$ the reverse is true (the larger the collision frequency the smaller the wavelength). Figure 9 shows that for angles larger than $89.75^{\circ}$ the larger the collision frequency the smaller the phase velocity for a given angle, whereas for smaller angles the reverse is true. Also there appears to be a minimum developing in the phase velocity in the range $89.3^{\circ} \leq \theta \leq 89.75^{\circ}$ as one decreases the collision frequency. Changing the electron and ion temperatures but keeping $T_{e}=T_{1}$ and correspondingly changing $v_{0}$ by the squáre root of this factor merely result in a corresponding decrease by the square root of the temperature factor in $k$, there being no decrease or change in shape of the growth rate versus $\theta$ (this result can be seen from the form of the dispersion relation (5)).

It should be pointed out that in the cases studied here the maximum growth rates occur for wavelengths that are in the range of tens of centimeters. This is much shorter than the 1to $10-\mathrm{m}$ irregularity wavelengths examined by the radar backscatter $(\lambda$ (irregularity) $=1 / 2 \lambda($ radar $)$ ) in studying the equatorial electrojet [Balsley and Farley, 1971]. One of the un-

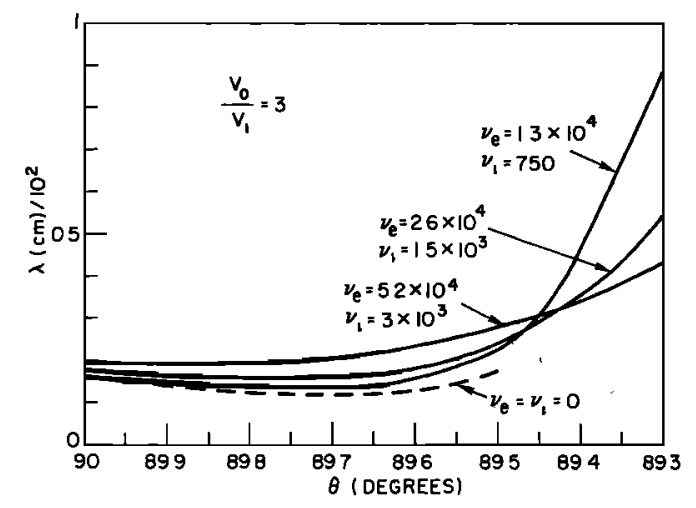

Fig. 8. Wavelength $\lambda$ versus angle $\theta$ corresponding to the unstable waves plotted in Figure 7. 


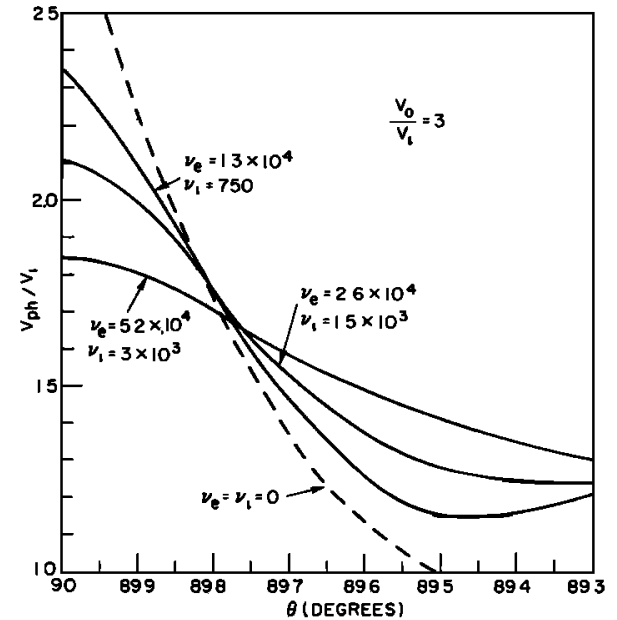

Fig. 9. Phase velocity in units of ion acoustic velocity $\left(v_{p h} / v_{l}\right)$ versus angle $\theta$ for unstable waves plotted in Figure 7 .

explained properties of the electrojet is that the waves that dominate the radar backscatter results have phase velocities around $v_{t}$ [Cohen and Bowles, 1967; Balsley and Farley, 1971]. The maximum growth rates that occur slightly off angle in Figure 1 (where $v_{0} / v_{t}=2.5-3$ ) show, in the frame where the ions are at rest, that the phase velocity is in the range 1.5-1.7vi (for $\theta=89.7^{\circ}-89.8^{\circ}$ ). Figure 3 shows the severe decrease in phase velocity as one departs from $90^{\circ}$. Indeed, for both $v_{0}=$ $2.5 v_{i}$ and $v_{0}=3 v_{i}$ there are off-angle modes with growth rates comparable to the rate at $90^{\circ}$ but with phase velocities much smaller than the velocity of the $90^{\circ}$ (exactly perpendicular) propagating wave. Also the most unstable wave at $89.3^{\circ}$ has a wavelength of $\sim 1 \mathrm{~m}$. In the lower drift velocity cases $v_{0} / v_{l}=2$ and 1.5 , the unstable waves approach the radar regime at $89.5^{\circ}$ and $89.7^{\circ}$, respectively, with phase velocities much lower than the velocity of the most unstable wave at $90^{\circ}$. For example, in the case of $v_{0} / v_{i}=2$ there is a certain flatness to the growth curve (Figure 1), and yet the phase velocity (Figure 3) decreases. In any case, for the parameters shown in Figure 1 the largest growth rate, whether it be at $90^{\circ}$ or slightly away from $90^{\circ}$, occurs for $\lambda$ in the range of tens of centimeters.

In the cases showing the effect of varying the density (Figure 4) for $v_{0} / v_{i}=3$ the growth rate maximizes off angle with $\lambda$ in the range of tens of centimeters and $v_{p h} / v_{l} \sim 1.7-1.9$ (Figure 6). In the case of $n_{0}=2.3 \times 10^{3} \mathrm{~cm}^{-3}$ the growth rate curve is fairly broad; yet the phase velocity of the most unstable wave at $90^{\circ}$ is $\approx 2.5 v_{i}$, whereas that at $89.3^{\circ}$ is $\approx 1.4 v_{i}$ (the growth rates differ by less than a factor of 2 ). Indeed, the growth rate at $90^{\circ}$ is comparable to that at $89.5^{\circ}$; yet the phase velocity at $90^{\circ}$ is $\approx 2.5 v_{i}$, whereas that at $89.5^{\circ}$ is $\approx 1.6 v_{i}$. The higher density growth rate curves are not quite as broad as the $n_{0}=2.3 \times$ $10^{\mathrm{s}} \mathrm{cm}^{-3}$ case, but the previous remarks apply to them as well. Moreover, comparing the growth rate at $90^{\circ}$ with a comparable off-angle value shows that the phase velocities in all these density cases differ by more than $25 \%$. Also we note that only in the case $n_{0}=2.3 \times 10^{3} \mathrm{~cm}^{-3}$ does one of the most unstable waves (at $89.3^{\circ}$ ) approach radar wavelengths.

In the case of our studies with collision frequencies a reduction $\left(\nu_{e}=1.3 \times 10^{4}, \nu_{t}=750\right)$ shows a more severe decrease in phase velocity. For example, the growth rate at $90^{\circ}$ is comparable to that at $89.6^{\circ}$, but the phase velocity is $2.35 v_{i}$ and $1.26 v_{i}$, respectively. Also at $89.3^{\circ}$ the most unstable wave approaches the radar wavelength regime. For the collision frequencies shown in Figure 7 the maximum growth rate occurs

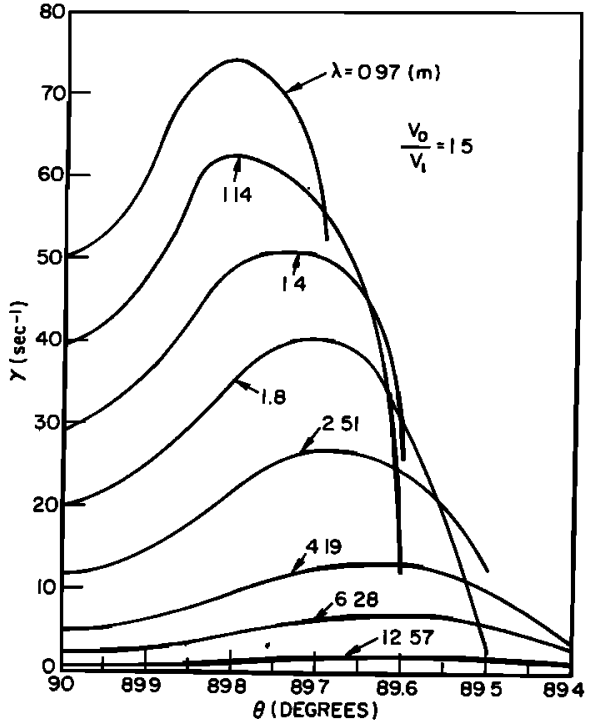

Fig. 10. Growth rate versus angle for irregularity wavelengths in the radar regime ( $\lambda$ plotted is one half the radar wavelength) with drift velocity $v_{0} / v_{t}=1.5$. All other parameters are the same as those in Figure 1.

for $\lambda \sim 10-20 \mathrm{~cm}$ (Figure 8). The higher collision frequency case results in a flattening of the growth rate curve (Figure 7), but it still results in a reasonable size decrease in the phase velocity (Figure 9). Maximal growth rates for all cases considered occur for waves whose phase velocity is $\sim 1.7 v_{i}$.

For completeness, we show in Figures 10-13 the growth rate and phase velocity versus angle for waves whose wavelengths are in the radar regime for drift velocities $v_{0} / v_{l}=1.5$ and 3 , the other parameters being the same as those in Figure 1. In both cases we see that the growth rates maximize slightly away from perpendicular to the magnetic field. It is also seen that the growth rates for these waves are for the most part considerably less than the maximum growth rates depicted in Figure 1. Only in the case $v_{0} / v_{t}=1.5$ for angles less than $89.8^{\circ}$ are the radar wavelength growth rates comparable to the maximum growth rates depicted in Figure 1. Figure 10 shows that the longer

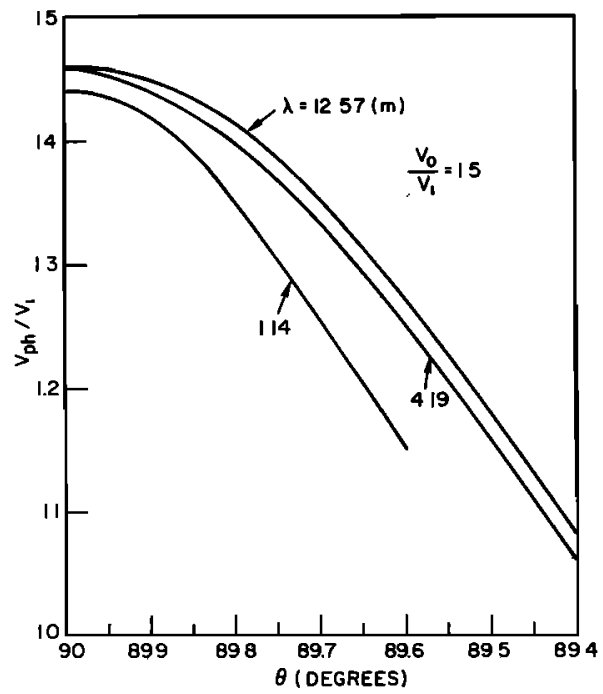

Fig. 11. Phase velocity in units of ion acoustic velocity $\left(v_{p h} / v_{l}\right)$ versus angle $\theta$ for representative radar wavelengths plotted in Figure 10. All wavelengths have not been shown because of the proximity of the curves. 


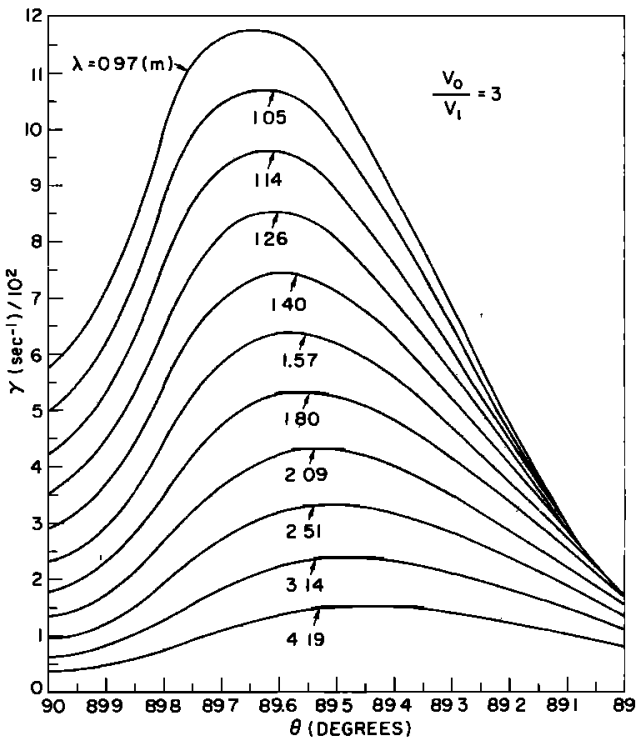

Fig. 12. Growth rate versus angle for irregularity wavelengths in the radar regime with $v_{0} / v_{t}=3$. All other parameters are the same as those in Figure 1.

radar wavelengths $(>4 \mathrm{~m}$ ) have broad maximums in growth between $89.4^{\circ}$ and $90^{\circ}$. For example, the case $\lambda=4.19 \mathrm{~m}$ shows that the growth rate at $90^{\circ}$ is comparable to that at $89.4^{\circ}$ (see Figure 10 ); yet the phase velocities are $1.46 v_{\imath}$ and $1.06 v_{1}$, respectively. (See Figure 11; note that only a few of the wavelengths represented in Figure 10 are shown in Figure 11 because of the proximity of the curves.) To be sure, the maximum growth rate occurs at $\sim 89.6^{\circ}$ (about $2 \frac{1}{2}$ times the growth rate at $\left.90^{\circ}\right)$, where the phase velocity is $1.25 v_{t}\left(1.46 v_{t}\right.$ at $\left.90^{\circ}\right)$. For the case of $\lambda=12.57 \mathrm{~m}$ the growth rate curve is even flatter. The maximum growth rates for the wavelengths depicted in Figure 10 occur for waves whose phase velocities are in the range $1.25-1.35 v_{i}$ and are down from the values at $90^{\circ}$. The shorter radar wavelengths have sharper peaks in growth rate, but even for the case $\lambda=0.97 \mathrm{~m}$ the growth rate at $90^{\circ}$ is comparable to that at $89.7^{\circ}$ and is only $50 \%$ below the peak at $89.8^{\circ}$. However, the phase velocity (Figure 11 ) at $90^{\circ}$ is $1.43 v_{i}$, whereas that at $89.7^{\circ}$ is $1.24 v_{i}$. In any case, phase velocities approaching what the radar sees are exhibited as one goes away from $90^{\circ}$.

Figure 12 shows the growth rate behavior of the radar wavelengths for larger drift velocity $\left(v_{0} / v_{l}=3\right)$. Once again the longer wavelengths show a broader maximum. For example, the curve for $\lambda=4.19 \mathrm{~m}$ has a maximum at $\sim 89.4^{\circ}$ (with phase velocity $\sim 2.04 v_{i}$; see Figure 13 ), which is about a factor of 4 greater than the growth rate at $90^{\circ}$. Moreover, the growth rate at $90^{\circ}$ is a factor of 2 smaller than that at $89^{\circ}$; yet the phase velocity at these two angles is $2.92 v_{l}$ and $1.38 v_{l}$, respectively (and still decreasing at $89^{\circ}$ ). The shorter wavelengths show sharper peaks. For example, the $\lambda=0.97 \mathrm{~m}$ curve (Figure 12) shows a maximum at $89.6^{\circ}\left(v_{p h} / v_{l} \approx 2 v_{i}\right)$, the growth rate at $90^{\circ}$ is comparable to that at $89.2^{\circ}$, and the phase velocities are $2.85 v_{i}$ and $1.28 v_{l}$, respectively (see Figure 13). The phase velocity at $89^{\circ}$ is $1.1 v_{i}$, whereas the growth rate is about $1 / 8$ that at $90^{\circ}$ and slightly less than $10 \%$ of the peak value at $89.6^{\circ}$.

We feel that we have presented here a relatively complete survey of the linear features of the type 1 electrojet instability in a homogeneous medium. It has been shown that off-angle $\left(k_{||} \neq 0\right)$ modes can be unstable for values in parameter space

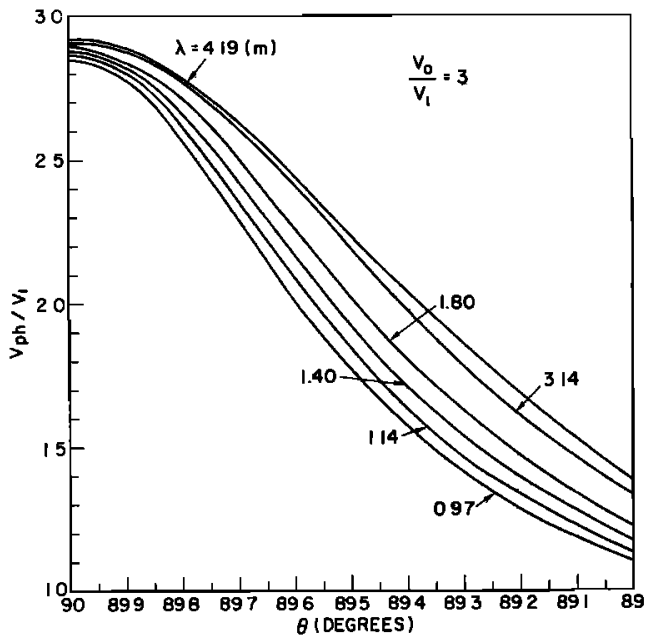

Fig. 13. Phase velocity in units of ion acoustıc velocity $\left(v_{p h} / v_{l}\right)$ versus angle for representative wavelengths plotted in Figure 12.

applicable to the electrojet regions and actually might dominate the linear stage in the higher drift velocity regimes. Actually, the linear growth rate of the radar wavelengths $(1-10 \mathrm{~m})$ always peaks slightly away from $90^{\circ}$ with decreasing phase velocity. Recent auroral radar observations have noted the effect of $k_{1 \mid} \neq 0$ modes ( $R$. Tsunoda, private communication, 1974). We expect that the survey presented can be of value in guiding experimentalists performing backscattering experiments in the equatorial electrojet.

Another important point is due to the fact that the observational features of the type 1 irregularities arise from a saturated or marginally stable nonlinear state. Since the $k_{||} \neq 0$ modes are of different physical nature, i.e., reactive or resistive-inductive modes, a stabilization mechanism different from the one stabilizing the purely resistive $k_{11}=0$ modes might be required. Any nonlinear scheme, whether it is resonance-broadening or mode-coupling, should be reexamined to determine its effect on the reactive modes. Before closing we should mention that the intriguing fact that these modes $\left(k_{11} \neq 0\right)$ have phase velocities closer to the ion sound speed seems to direct our research toward some nonlinear modecoupling scheme that can couple to these lower phase velocity waves. This would have the proper characteristics of the phase velocity of the waves seen by the radar for the type 1 electrojet instabilities. (The results of our studies showing unstable waves off angle with phase velocities approaching the ion sound speed are suggestive rather than definitive with respect to the radar measurements.) Indeed, many people have attempted to explain the observed radar spectrum with maximum intensity near $v_{l}$ with a host of nonlinear and linear theories (see, for example, Skadron and Weinstock [1969], Weinstock and Sleeper [1972], Rogister [1971], Sato [1972, 1973], Farley and Balsley [1973], Lee et al. [1974] and also the critique therein, and Kaw [1972]). However, to our knowledge, none of the above theories include $k_{11}$ effects (they consider the plane to be perpendicular to the magnetic field), and we are presently examining the nonlinear problem with this in mind,

\section{APPENDIX}

In order to see how the nature of the instability changes off angle we consider the following analysis. We can think of (6) as being the interaction of some weak beamlike structure (weak because $\omega \ll \omega_{p e}$ ) with the electrons (see, for example, 
Briggs [1964]). The right-hand side of (6) can then be thought of as an equivalent dielectric constant

$$
\begin{aligned}
& D(k, \omega)=1+\left(\frac{\omega_{p e}}{\Omega_{e}}\right)^{2} \frac{\omega+i \nu_{e}}{\omega}-\left(\frac{k_{\|}}{k}\right)^{2} \frac{\omega_{p e}^{2}}{\omega\left(\omega+i \nu_{\rho}\right)} \\
& =1+\left(\frac{\omega_{p e}}{\Omega_{e}}\right)^{2}\left(1+\frac{i \nu_{e}}{\omega}\right)-\left(\frac{k_{\|}}{k}\right)^{2} \frac{\omega_{p e}^{2}}{\omega^{2}+\nu_{e}{ }^{2}}\left(1-\frac{i \nu_{e}}{\omega}\right)
\end{aligned}
$$

therefore

$$
D(k, \omega)=\operatorname{Re} D+i \operatorname{Im} D
$$

with

$$
\begin{aligned}
& \operatorname{Re} D(k, \omega)=\frac{\omega_{p e}{ }^{2}}{\Omega_{e}{ }^{2}}-\left(\frac{k_{\|}}{k}\right)^{2} \frac{\omega_{p e}{ }^{2}}{\omega^{2}+\nu_{e}{ }^{2}}+1 \\
& \operatorname{Im} D(k, \omega)=\frac{\omega_{p e}{ }^{2}}{\Omega_{e}{ }^{2}} \frac{\nu_{e}}{\omega}+\left(\frac{k_{\|}}{k}\right)^{2} \frac{\omega_{p s}{ }^{2}}{\omega^{2}+\nu_{e}{ }^{2}} \frac{\nu_{\theta}}{\omega}
\end{aligned}
$$

Equation (6) is then for $\omega_{p e} \gg \Omega_{e}$

$$
\begin{aligned}
\frac{-2{\omega_{p i}}^{2}}{k^{2} v_{2}{ }^{2}} & {\left[1+\frac{\omega-\mathbf{k} \cdot \mathbf{v}_{0}+i v_{i}}{k v_{i}} Z\left(\frac{\omega-\mathbf{k} \cdot \mathbf{v}_{0}+i \nu_{i}}{k v_{\imath}}\right)\right] } \\
\cdot & {\left[1+\frac{i \nu_{i}}{k v_{i}} Z\left(\frac{\omega-\mathbf{k} \cdot v_{0}+i \nu_{2}}{k v_{\imath}}\right)\right]^{-1}=\frac{\omega_{p e}{ }^{2}}{\Omega_{e}{ }^{2}} } \\
\cdot & \left\{\left[1-\left(\frac{k_{\|}}{k}\right)^{2} \frac{\Omega_{e}{ }^{2}}{\omega^{2}+\nu_{0}{ }^{2}}\right]+\frac{i \nu_{e}}{\omega}\left(1+\left(\frac{k_{\|}}{k}\right)^{2} \frac{\Omega_{e}{ }^{2}}{\omega^{2}+\nu_{e}{ }^{2}}\right)\right\}
\end{aligned}
$$

Then

$$
\begin{aligned}
\epsilon_{i}(k, \omega)=\left[1-\left(\frac{k_{\|}}{k}\right)^{2} \frac{\Omega_{e}{ }^{2}}{\omega^{2}+\nu_{e}{ }^{2}}\right] \\
+\frac{i \nu_{e}}{\omega}\left[1+\left(\frac{k_{\| 1}}{k}\right)^{2} \frac{\Omega_{e}{ }^{2}}{\omega^{2}+\nu_{e}{ }^{2}}\right]
\end{aligned}
$$

In order to examine the physics of the instability we can take the cold ion approximation. Then the ions behave like a beam with frequency $\Omega_{H}=\left(m_{e} / m_{i}\right)^{1 / 2} \Omega_{e}=\left(\Omega_{e} \Omega_{l}\right)^{1 / 2}$. Equation (11) is then

$$
\begin{aligned}
& \frac{\Omega_{H}{ }^{2}}{\left(\omega-\mathbf{k} \cdot \nabla_{0}\right)\left(\omega-\mathbf{k} \cdot \nabla_{0}+i \nu_{i}\right)} \\
& =1-\left(\frac{k_{\|}}{k}\right)^{2} \frac{\Omega_{e}{ }^{2}}{\left(\omega^{2}+\nu_{e}{ }^{2}\right)}+i \frac{\nu_{e}}{\omega}\left[1+\left(\frac{k_{\|}}{k}\right)^{2} \frac{\Omega_{e}{ }^{2}}{\omega^{2}+\nu_{e}^{2}}\right] \\
& =\operatorname{Re} D_{1}+i \operatorname{Im} D_{1}
\end{aligned}
$$

If $\left|\operatorname{Re} D_{1}\right| \gg\left(\operatorname{Im} D_{1}\right)$, then instability occurs for $\operatorname{Re} D_{1}<0$, and we then have strong reactive amplification (negative dielectric constant). When $\operatorname{Re} D_{1}>0$, one can have only resistive type amplification. The slow wave (which carries negative energy) grows while the fast wave decays. The resistive medium extracts energy from the ion drift and transfers it to the slow wave.

For $\theta=\pi / 2, k_{11}=0$ (Farley-Buneman branch), and in (12), $\operatorname{Re} D_{1}>0$ always. In this case and for more general angles such that

$$
1-\left(\frac{k_{11}}{k}\right)^{2} \frac{\Omega_{e}{ }^{2}}{\omega^{2}+\nu_{e}{ }^{2}}>0
$$

the instability is always of the resistive type. In this case,

$$
\Omega_{H}{ }^{2} /\left[\left(\omega-\mathrm{k} \cdot \mathrm{v}_{0}\right)\left(\omega-\mathrm{k} \cdot \mathrm{v}_{0}+i \nu_{l}\right)\right]=1+i\left(\nu_{e} / \omega\right)
$$

For $\nu_{e} / \omega_{r} \gg 1$ we find the Farley-Buneman limit with $\omega_{r}=$ $\left(\alpha \mathbf{k} \cdot \mathrm{v}_{0}\right) /(1+\alpha)$, where $\alpha=\nu_{e} \nu_{i} / \Omega_{H}{ }^{2}$ (note that this is the solution to the Buneman limit for $\omega_{r}$ in the electron rest frame; see, for example, Rogister and D'Angelo [1970] and Sudan et al. [1973]); if $\nu_{e} / \omega_{r} \ll 1$, we find the solution of Lee et al. [1971] with $\omega_{r}=\Omega_{H}+\mathbf{k} \cdot \mathbf{v}_{0}$.

The important point is that for $\left(k_{||} / k\right)^{2}\left[\Omega_{e}{ }^{2} /\left(\omega^{2}+\nu_{e}{ }^{2}\right)\right]>1$ there is a strong reactive instability, since $\operatorname{Re} D_{1}<0$. This is equivalent to the modified two-stream instability [McBride et al., 1972] with the addition of an imaginary part from the term Im $D_{1}$. In this limit and for $\gamma>\nu_{i},(12)$ becomes

$$
\frac{\Omega_{H}{ }^{2}}{\left(\omega-\mathbf{k} \cdot \nabla_{0}\right)^{2}}=-\left(\frac{k_{\|}}{k}\right)^{2} \frac{\Omega_{e}{ }^{2}}{\omega^{2}+\nu_{e}{ }^{2}}\left(1-\frac{i \nu_{e}}{\omega}\right)
$$

with the condition that $\omega_{r} / k_{||} \gg v_{e}$ be satisfied. If we drop the term $\nu_{e} / \omega$ by assuming that $\nu_{e} / \omega<1$, the results are known and are given by $\gamma \sim \omega_{r} \sim \mathrm{k} \cdot \mathrm{v}_{0} \sim 1 / 2\left(k_{||} / k\right) \Omega_{H}$. Thus the simplified analysis showing the change in nature of the instability off angle is completed.

Acknowledgments. This work was supported by ONR and ARPA. The Editor thanks D. T. Farley and K. Lee for their assistance in evaluating this paper.

\section{REFERENCES}

Balsley, B. B., and D. T. Farley, Radar studies of the equatorial electrojet at three frequencies, J. Geophys. Res., 76, 8341, 1971.

Bhatnagar, P. L., E. P. Gross, and M. Krook, A model for collision processes in gases, 1, Small amplitude processes in charged and neutral one-component systems, Phys. Rev., 94, 511, 1954.

Briggs, R. J., Electron Stream Interaction With Plasmas, pp. 48-60, MIT Press, Cambridge, Mass., 1964.

Buneman, $O$., Excitation of field aligned sound waves by electron streams, Phys. Rev. Lett., 10, 285, 1963.

Cohen, R., and K. L. Bowles, Secondary irregularities in the equatorial electrojet, J. Geophys. Res., 72, 885, 1967.

Farley, D. T., Jr., The two-stream instability as a source of irregularities in the ionosphere, Phys. Rev. Lett., 10, 279, 1963a.

Farley, D. T., Jr., A plasma instability resulting in field-aligned irregularities in the ionosphere, J. Geophys. Res., 68, 6083, $1963 \mathrm{~b}$.

Farley, D. T., and B. B. Balsley, Instabilities in the equatorial electrojet, J. Geophys. Res., 78, 227, 1973.

Fried, B. D., and S. D. Conte, The Plasma Dispersion Function, pp. 1-3, Academic, New York, 1961.

Kaw, P. K., Wave propagation effects on observation of irregularities in the equatorial electrojet, J. Geophys. Res., 77, 1323, 1972.

Krall, N. A.. and P. C. Liewer, Low-frequency instabilities in magnetic pulses, Phys. Rev., Sect. A, 4, 2094, 1971.

Lee, K., and C. F. Kennel, Effects of propagation parallel to the magnetic field on the type 1 electrojet irregularity instability, Planet. Space Sci., 21, 1339, 1973.

Lee, K., C. F. Kennel, and J. M. Kindel, High-frequency Hall current instability, Radio Sci., 6, 209, 1971.

Lee, K., C. F. Kennel, and F. V. Coroniti, On the marginally stable saturation spectrum of unstable type 1 equatorial electrojet irregularities, J. Geophys. Res., 79, 249, 1974.

McBride, J. B., E. Ott, J. P. Boris, and J. H. Orens, Theory and simulation of turbulent heating by the modified two-stream instability, Phys. Fluids, 15, 2367, 1972.

Rogister, A., Nonlinear theory of 'type 1' irregularities in the equatorial electrojet, J. Geophys. Res., 76, 7754, 1971.

Rogister, A., and N. D'Angelo, Type 2 irregularities in the equatorial electrojet, J. Geophys. Res., 75, 3879, 1970.

Sato, T., Stabilization of the two-stream instability in the equatorial electrojet, Phys. Rev. Lett., 28, 732, 1972.

Sato, T., Unified theory of type 1 and type 2 irregularities in the equatorial electrojet, J. Geophys. Res., 78, 2232, 1973.

Schmidt, M. J., and S. P. Gary, Density gradients and the FarleyBuneman instability, J. Geophys. Res., 78, 8261, 1973. 
Skadron, G., and J. Weinstock, Nonlinear stabilization of a twostream plasma instability in the ionosphere, J. Geophys. Res., 74, $5113,1969$.

Sudan, R. N., J. Akinrimisi, and D. T. Farley, Generation of smallscale irregularities in the equatorial electrojet, J. Geophys. Res., 78, $240,1973$.
Weinstock, J., and A. Sleeper, Nonlinear saturation of 'type l' irregularities in the equatorial electrojet, J. Geophys. Res., 77. 3621, 1972.

(Received January 29, 1974; accepted September 13, 1974.) 